\title{
Botulinumtoxin bei laryngealer Dystonie: Alter und Geschlecht entscheidend?
}

\section{Botulinumtoxin-Injektionen werden seit 20 Jahren effektiv in der Therapie der laryngealen Dystonie eingesetzt. Zur Dosierung existiert jedoch kein einheitlicher Algorithmus. Wissenschaftler prüften nun, inwieweit Alter und Geschlecht hierbei eine Rolle spielen.}

E rgebnisse früherer kleinerer Untersuchungen ließen vermuten, dass sich die effektiven Dosierungen von Botulinumtoxin in der Therapie der laryn-

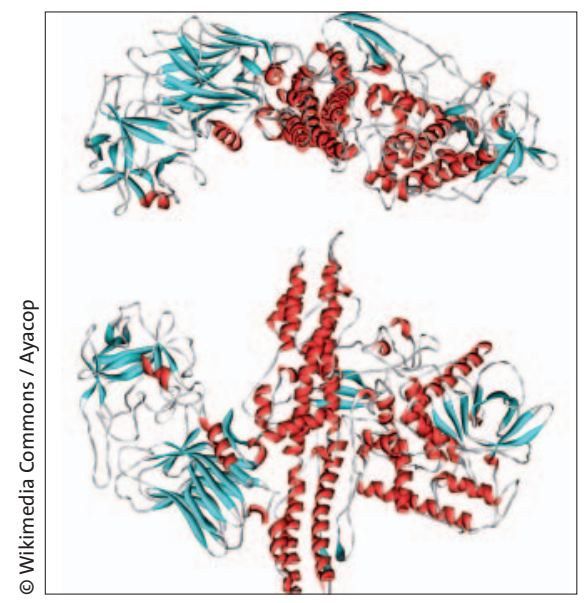

3-D-Modell von Botulinumtoxin A gealen Dystonie mit zunehmendem Alter ändern und dass Männer im Durchschnitt eine höhere Dosis benötigen als Frauen. Ein internationales Forscherteam wertete die Daten von 155 Patienten (50 Männer, 105 Frauen) aus, die zwischen 1991 und 2008 an der University of Washington in Seattle wegen einer laryngealen Dystonie vom Adduktor-Typ behandelt worden waren. Das durchschnittliche Alter betrug bei den männnlichen Probanden 50,6 Jahre, bei den weiblichen lag es bei 54,4 Jahren.

Folgende Parameter gingen in die Studie ein: Geschlecht, Alter zu Beginn der Therapie, Dosierung von Botulinumtoxin sowie die Patientenangabe zur Wirkdauer der Injektion. Bezüglich Dosierung und Wirkdauer wurden erst die Angaben ab der sechsten Injektion einbezogen, um mögliche Effekte der Dosisfindung bei den ersten fünf Injekti- onen auszuschließen. Die Injektionen waren ausschließlich in den M. thyroarytenoideus erfolgt.

Die mittlere Dosis von Botulinumtoxin lag bei den Männern bei 1,85 $\pm 0,84 \mathrm{U}$, bei Frauen bei $2,15 \pm 1,40$ U. Die durchschnittliche Wirkdauer der Injektionen betrug bei Männern 12,8 \pm 7,7 Wochen, bei Frauen lag sie bei 13,9 \pm 7,3 Wochen. Für Patienten im Alter von unter 50 Jahren lag die mittlere Dosierung bei $1,87 \pm$ $1,00 \mathrm{U}$ und die Wirkdauer bei $13,7 \pm 7,4$ Wochen, für die über 50-Jährigen waren die entsprechenden Werte 2,20 $\pm 1,41 \mathrm{U}$ beziehungsweise 13,5 \pm 7,4 Wochen. Keiner der Unterschiede war statistisch signifikant.

Fazit: Entgegen der Ausgangshypothese der Studie waren Dosis und Wirkdauer weder vom Geschlecht noch vom Alter abhängig. Die Wissenschaftler vermuten, dass andere Faktoren die erforderliche effektive Dosis von Botulinumtoxin bei laryngealer Dystonie beeinflussen. mlg

Vasconcelos $S$ et al. Influence of age and gender on dose and effectiveness of botulinum toxin for laryngeal dystonia.

Laryngoscope 2009;119:2004-7

\section{Kombinierte OP bei großen Speichelsteinen}

Bei Sialolithiasis mit großen Konkrementen wird oft die gesamte Speicheldrüse reseziert. US-amerikanische Forscher werteten die Ergebnisse von Operationen aus, bei denen kombiniert endoskopisch und mit offenem Zugang große Speichelsteine entfernt wurden.

U S-amerikanische HNO-Ärzte analysierten die Daten von Patienten, die sich aufgrund großer Speichelsteine ( $>4$ $\mathrm{mm}$ in der Glandula submandibularis bzw. > $3 \mathrm{~mm}$ in der Glandula parotis) einer kombinierten Operation unterzogen. Bei dem Eingriff wurde der Stein zunächst endoskopisch lokalisiert, dann fixiert und wenn möglich mit einem Drahtkorb umschlossen. Die Operateure entfernten den Stein nach Eröffnung des betroffenen Drüsengangs über einen offenen Zugang - intraoral bei submandibulären oder nach außen bei parotidealen Steinen. Eine distale Endoskopie sicherte die Entfernung weiterer Fragmente oder
Steine. Bei 19 Patienten mit symptomatischer Sialolithiasis wurden auf diese Art 20 Speichelsteine entfernt. 70\% waren Steine der Glandula submandibularis, ebenfalls in $70 \%$ der Fälle handelte es sich um linksseitig gelegene Steine.

In 18 von 20 Fällen (90\%) konnten die Konkremente ohne größere Komplikationen entfernt werden. Kleinere OPKomplikationen traten in vier Fällen (20\%) auf: Bei zwei Patienten (10\%) mussten die Steine mit der Speicheldrüse herausgenommen werden. Ein Patient litt an einer vorübergehenden Paresthesie des N. lingualis, bei einem weiteren Patient schwoll die betroffene Speicheldrüse an; diese Schwellung ging jedoch nach konservativer Behandlung zurück. Im weiteren Beobachtungsverlauf berichtete nur ein Patient über weitere Beschwerden im Bereich der operierten Speicheldrüse, die aber ebenfalls unter konservativen Maßnahmen nachließen. Die Operationsdauer lag bei 90 Minuten für die Steinentfernung aus der Glandula submandibularis. Für den Eingriff bei der Parotis benötigten die Operateure 133 Minuten.

Fazit: Das kombinierte Vorgehen aus endokopischem und offenem Eingriff ermöglicht es, große Speichelsteine ohne Resektion der gesamten Drüse zu entfernen. Die Erfolgsquote lag in dieser retrospektiven Analyse bei 90\%.

af

Walvekar RR et al. Combined approach technique for the management of large salivary stones. Laryngoscope 2009;119:1125-9 